\title{
A nationwide survey concerning practices in pessary use for pelvic organ prolapse in The Netherlands: identifying needs for further research
}

\author{
Joost Velzel ${ }^{1,2}$ • Jan Paul Roovers ${ }^{1}$ - C H Van der Vaart ${ }^{3}$ - Bart Broekman ${ }^{4}$. \\ Astrid Vollebregt $^{2} \cdot$ Robert Hakvoort $^{2}$
}

Received: 16 December 2014 / Accepted: 16 March 2015 / Published online: 11 June 2015

(C) The Author(s) 2015. This article is published with open access at Springerlink.com

\begin{abstract}
Introduction and hypothesis To identify practice variation in management of patients with a vaginal pessary for pelvic organ prolapse (POP).

Methods A nationwide survey was sent to all Dutch gynecologists with a special interest in urogynecology.

Results The response rate was $59 \%$. Of the respondents, $13 \%$ had a written protocol for pessary treatment in their department. Pessary treatment was proposed by $69 \%$ of respondents as a treatment option. Counseling about side effects varied. All respondents provided information concerning the possibility of serious vaginal discharge. Concerning this side effect, $15 \%$ of the respondents stated that it occurs in $5-20 \%$ of patients, $27 \%$ that it occurs in $20-40 \%$ of patients, and $57 \%$ that it occurs in more than $40 \%$ of patients. Another item concerned counseling for the likelihood of vaginal blood loss. All respondents provided information concerning the possibility of vaginal blood loss. Concerning this side effect, $53 \%$ of the respondents stated that it occurs in $5-20 \%$ of patients, $33 \%$ that it occurs in $20-40 \%$, and $14 \%$ that it occurs in more than $40 \%$ of patients. Follow-up after initial placement
\end{abstract}

Joost Velzel

j.velzel@amc.nl

1 Department of Obstetrics and Gynecology, Academic Medical Center Amsterdam, Meibergdreef 9, 1105

AZ Amsterdam, The Netherlands

2 Department of Obstetrics and Gynecology, Spaarne Ziekenhuis, Hoofddorp, The Netherlands

3 Department of Obstetrics and Gynecology, University Medical Centre, Utrecht, The Netherlands

4 Department of Obstetrics and Gynecology, Sint Franciscus Gasthuis, Rotterdam, The Netherlands was done by $69 \%$ of the respondents at $2-6$ weeks, by $2 \%$ at 8 weeks, and by $29 \%$ at 12 weeks or more. Most ( $98 \%$ ) of the respondents extended the interval between visits when the patient had no complaints, and $96 \%$ of the respondents reported that they routinely instruct patients about selfmanagement.

Conclusions Pessaries are suggested as a treatment option by a majority of gynecologists, but practice varies widely. We consider that the variation in practice is due to a lack of available protocols and lack of evidence.

Keywords Prolapse $\cdot$ Pessary $\cdot$ Survey $\cdot$ Prolapse management

\section{Introduction}

Pelvic organ prolapse (POP) is a common condition in adult women [1]. For the relief of symptoms related to POP conservative treatment options include life-style advice, pelvic physiotherapy and pessary treatment [2-4]. The aim of pessary treatment is to restore the anatomy of the visceral organs of the pelvic cavity by inserting a device into the vagina. The reported subjective cure rates vary between $60 \%$ and $80 \%[5$, 6]. Many patients prefer this treatment over surgery, as it is unlikely to cause serious morbidity and normal activities can be continued [7-10]. Successful pessary fitting and long-term success have been documented in up to $75 \%$ of women with symptomatic POP [11-14]. The reason for the high proportion $(80-85 \%)$ of gynecologists placing pessaries may be the ease with which they can be inserted and removed, their efficacy and the low complication rate $[15,16]$.

Although large groups of women receive these devices, a national guideline regarding POP was not available in The Netherlands at the time of our survey. The proportion of 
patients made aware of this treatment option, which patients are most eligible for this treatment, and how long follow-up intervals should be were unknown. Ignorance concerning these issues may result in practice variation and suboptimal treatment of patients. Therefore, the aim of the present survey was to identify current practice and variation in practice concerning treatment with vaginal pessaries for POP.

\section{Materials and methods}

An invitation to participate was sent to all 151 members of the Dutch working party for urogynecology of the Dutch College for Obstetrics and Gynecology. This membership represents $15.4 \%$ of the 981 Dutch gynecologists. We expected all members of the Dutch working party for urogynecology to be familiar with the indications for and performance of pessary treatment. Participants were asked to answer the survey using an online system (SurveyMonkey ${ }^{\circledR}$ ). A reminder was sent to those who had not responded 4 weeks after the first request. Nonresponders were sent a third request 4 weeks after the second request.

The questionnaire was developed specifically for the survey based on previous questionnaires $[15,16]$ and by a panel of four experts in urogynecology. The questionnaire included both multiple choice and open questions addressing the following topics: characteristics of gynecologists and hospitals, selection of patients, follow-up management and counseling patients about side effects, effectiveness and the option of selfmanagement. We asked the respondents to answer as if considering a patient without a previous pessary fitting trial. The full questionnaire can be found in Table 5 in the Appendix. Ethics review board approval was not applied for.

Data from the completed questionnaires were used to calculate frequencies and percentages of respondents answering per question per topic. Descriptive data analysis was performed using SPSS 19.0 (SPSS Statistics UK, SPSS Inc., Chicago, IL).

\section{Results}

After three requests, a total of 91 of 151 gynecologists (59\%) in 63 of 80 hospitals ( $79 \%$ ) had responded. In $98 \%$ of the responding hospitals a gynecologist with a special interest in urogynecology was employed. A written protocol for indication, insertion and follow-up of pessary treatment was available in $13 \%$ (Table 1 ).

Pessary placement for the treatment of POP was proposed by $69 \%$ of respondents always, by $17 \%$ sometimes, and by $14 \%$ never. Prolapse of the anterior compartment and apical compartment were considered the most suitable indications for pessary treatment $(99 \%$ and $96 \%$ of the respondents,
Table 1 Characteristics of the hospitals of responding gynecologists

\begin{tabular}{lll}
\hline & General hospital & $\begin{array}{l}\text { No. (\%) of } \\
\text { respondents }\end{array}$ \\
\hline Type of hospital & Teaching hospital & $55(29)$ \\
& Academic hospital & $9(10)$ \\
& Private practice & $1(1)$ \\
New patients with vaginal & $0-400$ & $24(26)$ \\
prolapse annually & $401-800$ & $46(51)$ \\
& $>800$ & $21(23)$ \\
Vaginal prolapse surgery & $0-100$ & $14(15)$ \\
procedures annually & $101-200$ & $52(57)$ \\
& $>200$ & $25(28)$ \\
Gynecologist with special & Yes & $89(98)$ \\
interest urogynecology & No & $2(2)$ \\
Existence of written protocol & Yes & $12(13)$ \\
for pessary use & No (but consensus & $54(59)$ \\
& among caregivers) & $25(27)$ \\
\hline
\end{tabular}

respectively). Concerning the decision to start initial treatment with a pessary, $62 \%$ of the respondents stated that they were not influenced by the stage of prolapse, and $36 \%$ stated that patient age was a decisive factor, with younger patients being less likely to receive a pessary (Table 2).

Table 3 shows the protocols and practices of gynecologists concerning follow-up after initial placement. The interval between initial placement and first follow-up varied from 2 weeks to 16 weeks. The first follow-up visits were at 2 weeks (17\% of respondents), 3 weeks (15\%), 4 weeks (14\%),

Table 2 Selection of patients

\begin{tabular}{|c|c|c|}
\hline & & $\begin{array}{l}\text { No. }(\%) \text { of } \\
\text { respondents }\end{array}$ \\
\hline \multirow{3}{*}{$\begin{array}{l}\text { Standard information } \\
\text { about the option of } \\
\text { a pessary }\end{array}$} & Yes & $63(69)$ \\
\hline & Occasionally & $15(17)$ \\
\hline & No & $13(14)$ \\
\hline \multirow{7}{*}{$\begin{array}{l}\text { Type of prolapse thought } \\
\text { to be most suitable for } \\
\text { pessary }^{\mathrm{a}}\end{array}$} & Prolapse anterior compartment & $88(99)$ \\
\hline & Prolapse middle compartment & $85(96)$ \\
\hline & $\begin{array}{l}\text { Prolapse posterior } \\
\text { compartment }\end{array}$ & $9(11)$ \\
\hline & Stress incontinence & $11(12)$ \\
\hline & Urge incontinence & $8(9)$ \\
\hline & Constipation & $0(0)$ \\
\hline & Obstructed defecation & $26(29)$ \\
\hline \multirow{2}{*}{$\begin{array}{l}\text { Influence of prolapse } \\
\text { stage on decision }\end{array}$} & Yes & $35(38)$ \\
\hline & No & $56(62)$ \\
\hline \multirow{2}{*}{$\begin{array}{l}\text { Influence of patient age } \\
\text { on decision }\end{array}$} & Yes & $33(36)$ \\
\hline & No & $58(64)$ \\
\hline
\end{tabular}

${ }^{\mathrm{a}}$ More answers possible 
Table 3 Follow up

\begin{tabular}{|c|c|c|}
\hline & & $\begin{array}{l}\text { No. }(\%) \text { of } \\
\text { respondents }\end{array}$ \\
\hline \multirow{7}{*}{$\begin{array}{l}\text { First follow-up visit } \\
\quad \text { (weeks) }\end{array}$} & 2 & $15(17)$ \\
\hline & 3 & $14(15)$ \\
\hline & 4 & $13(14)$ \\
\hline & 6 & $21(23)$ \\
\hline & 8 & $2(2)$ \\
\hline & 12 & $21(23)$ \\
\hline & 16 & $5(6)$ \\
\hline \multirow{5}{*}{$\begin{array}{l}\text { Professional responsible } \\
\text { for first follow-up visit }\end{array}$} & Same caregiver & $30(33)$ \\
\hline & $\begin{array}{l}\text { Same caregiver, later general } \\
\text { practitioner }\end{array}$ & $48(53)$ \\
\hline & $\begin{array}{l}\text { Same caregiver or general } \\
\text { practitioner }\end{array}$ & $1(1)$ \\
\hline & Specialist nurse & $1(1)$ \\
\hline & Patient wishes & $11(12)$ \\
\hline \multirow{3}{*}{$\begin{array}{l}\text { Timing of follow-up visits } \\
\text { after initial placement }{ }^{\mathrm{a}}\end{array}$} & Same interval continued & $15(16)$ \\
\hline & Shorter intervals if complaints & $27(30)$ \\
\hline & $\begin{array}{l}\text { Longer intervals if no } \\
\text { complaints }\end{array}$ & $90(99)$ \\
\hline \multirow{4}{*}{$\begin{array}{l}\text { Prescription of estrogens } \\
\quad \text { (oral and vaginal) }\end{array}$} & Yes & $12(13)$ \\
\hline & $\begin{array}{l}\text { When indicated (vaginal } \\
\text { atrophy) }\end{array}$ & $66(73)$ \\
\hline & $\begin{array}{l}\text { When indicated (other than } \\
\text { atrophy) }\end{array}$ & $8(9)$ \\
\hline & No & $5(5)$ \\
\hline
\end{tabular}

${ }^{\mathrm{a}}$ More answers possible

6 weeks (23\%), 8 weeks (2\%), 12 weeks $(23 \%)$, and 16 weeks $(6 \%)$.

Regarding the first follow up visit, 78 respondents (86\%) stated that it was carried out by a gynecologist, $2 \%$ delegated this care to either the general practitioner (GP) or a nurse practitioner, and $12 \%$ stated that the patient could choose between the above professionals. If there were no patient complaints, $99 \%$ of respondents increased the interval between visits. Estrogens were prescribed routinely by $13 \%$ of respondents, only when vaginal atrophy was present by $73 \%$, and when indications other than atrophy, for example irritation, were present by $9 \% ; 5 \%$ of respondents never prescribed estrogens.

Counseling about side effects varied. All respondents provided information concerning the possibility of serious vaginal discharge. Concerning this side effect, $15 \%$ of the respondents stated that it occurs in $5-20 \%$ of patients, $27 \%$ that it occurs in $20-40 \%$ of patients, and $57 \%$ that it occurs in more than $40 \%$ of patients. Another item concerned counseling for the likelihood of vaginal blood loss. All respondents provided information concerning the possibility of vaginal blood loss. Concerning this side effect, $53 \%$ of the respondents stated that it occurs in $5-20 \%$ of patients, $33 \%$ that it occurs in $20-40 \%$, and $14 \%$ that it occurs in more than $40 \%$ of patients. Concerning the success rates of pessary treatment, $5 \%$ of respondents informed patients that pessary treatment is successful in only $5-20 \%$ of patients, $46 \%$ that it is successful in $20-50 \%$ of patients, and $48 \%$ that it is successful in more than $50 \%$ of patients. The likelihood of eventually receiving surgical treatment following failure of pessary treatment was stated to be $0-25 \%$ by $24 \%$ of respondents, $25-50 \%$ by $56 \%$ and more than $50 \%$ by $20 \%$. Of the respondents who proposed pessaries as a treatment option, $96 \%$ informed the patient about the possibility of selfmanagement (Table 4).

\section{Discussion}

A nationwide survey was performed to quantify the variation among gynecologists in the practice of pessary treatment in women with symptomatic POP. A low percentage (only $13 \%$ ) of respondents stated that they had a written protocol available in their department. A relatively high percentage (69\%) of respondents routinely suggested pessary treatment for POP. A considerable variation was found in counseling about vaginal discharge and vaginal blood loss as possible side effects. Furthermore, the intervals between placement and the first follow-up visit varied greatly. A majority of the respondents reported that they routinely instruct the patient about selfmanagement.

Before discussing these results in more detail, we address some limitations of this study. A criticism might be that the $59 \%$ response rate did not reflect overall clinical practice. However, the respondents represented $79 \%$ of Dutch departments. Failure of a proportion of potential participants to respond means that trends that could have been recognized were missed. There could have been a reporting bias favoring younger potential participants due to the online survey tool used. However, it is not clear how this could have affected the results. As previous surveys on this topic had response rates of $21 \%$ [15] and $55 \%$ [16] we consider that the response rate can be regarded as high. Another possible limitation was the way in which questions were formulated. To be able to obtain answers that could be more easily analyzed multiple choice questions were mainly used, which may not always have reflected actual practice. Unfortunately, this is inherent in the survey format.

Also GPs contribute to this type of care, estimated in The Netherlands to be around $20 \%$ of the total amount of care, but this group was not interviewed. There is no expertise in pessary treatment for POP among other groups in The Netherlands, for example nurse practitioners. It would have been interesting to perform a similar survey among GPs to gain an insight into their use of pessaries in the management of POP. Because of the relatively small contribution of GPs to this care we decided not to include GPs in the study. 
Table 4 Patient information and self-management

\begin{tabular}{|c|c|c|c|}
\hline & & & $\begin{array}{l}\text { No. }(\%) \text { of } \\
\text { respondents }\end{array}$ \\
\hline \multirow[t]{6}{*}{ Likelihood of side effects of pessary use } & \multirow[t]{3}{*}{ Vaginal discharge } & $5-20 \%$ & $14(15)$ \\
\hline & & $20-40 \%$ & $25(27)$ \\
\hline & & $>40 \%$ & $52(57)$ \\
\hline & \multirow[t]{3}{*}{ Vaginal blood loss } & $5-20 \%$ & $48(53)$ \\
\hline & & $20-40 \%$ & $30(33)$ \\
\hline & & $>40 \%$ & $13(14)$ \\
\hline \multirow{4}{*}{$\begin{array}{l}\text { Likelihood of surgical treatment for POP } \\
\text { after pessary treatment }\end{array}$} & \multicolumn{2}{|l|}{$0-25 \%$} & $22(24)$ \\
\hline & \multicolumn{2}{|l|}{$25-50 \%$} & $51(56)$ \\
\hline & \multicolumn{2}{|l|}{$50-75 \%$} & $17(19)$ \\
\hline & \multicolumn{2}{|l|}{$75-100 \%$} & $1(1)$ \\
\hline \multirow[t]{4}{*}{ Likelihood of pessary extrusion } & \multicolumn{2}{|l|}{$5-15 \%$} & $36(40)$ \\
\hline & \multicolumn{2}{|l|}{$15-30 \%$} & $45(49)$ \\
\hline & \multicolumn{2}{|l|}{$30-50 \%$} & $8(9)$ \\
\hline & \multicolumn{2}{|l|}{$>50 \%$} & $2(2)$ \\
\hline \multirow{3}{*}{$\begin{array}{l}\text { Likelihood of that pessary treatment } \\
\text { will be effective/satisfactory }\end{array}$} & \multicolumn{2}{|l|}{$5-20 \%$} & $5(5)$ \\
\hline & \multicolumn{2}{|l|}{$20-50 \%$} & $42(46)$ \\
\hline & \multicolumn{2}{|l|}{$>50 \%$} & $44(48)$ \\
\hline \multirow{3}{*}{$\begin{array}{l}\text { Gynecologist giving advice } \\
\text { about self-management }\end{array}$} & \multicolumn{2}{|l|}{ Always } & $46(51)$ \\
\hline & \multicolumn{2}{|l|}{ Regularly } & $41(45)$ \\
\hline & \multicolumn{2}{|l|}{ No } & $4(4)$ \\
\hline \multirow{3}{*}{$\begin{array}{l}\text { Gynecologists giving instructions } \\
\text { about self-management }\end{array}$} & \multicolumn{2}{|l|}{ Yes, always } & $48(53)$ \\
\hline & \multicolumn{2}{|l|}{ Regularly } & $39(43)$ \\
\hline & \multicolumn{2}{|l|}{ No } & $4(4)$ \\
\hline \multirow{2}{*}{$\begin{array}{l}\text { Patients successful in self-management } \\
\text { returning to outpatient clinic }\end{array}$} & \multicolumn{2}{|l|}{ Yes } & $36(40)$ \\
\hline & \multicolumn{2}{|l|}{ No } & $55(60)$ \\
\hline
\end{tabular}

Furthermore, we recognize that this survey only dealt with daily practice in The Netherlands. Similar surveys in more countries could generate information of more clinical value.

The study clearly demonstrated that a considerable proportion (69\%) of gynecologists provide informs about the possibility of pessary treatment. The available literature clearly indicates that a high proportion of patients become long-term users with a high patient satisfaction $[10,13,17]$. This may be a result of the high percentage of patients who receive information about this treatment. In general, respondents considered pessary treatment for prolapse of the anterior and apical compartments as more successful than pessary treatment for prolapse of the posterior compartment. In literature, there is no definitive evidence that pessary use for posterior compartment prolapse is not as successful as pessary treatment for anterior wall prolapse [12, 18, 19]. The two largest studies have demonstrated $[12,18]$ no difference in the success of pessary treatment in relation to the type and severity of prolapse, and conclude that patients should not be selected for pessary treatment on the basis of the type of prolapse.

Information given to patients about adverse effects of pessary treatment varied to a great extent and the information that was given about the occurrence of side effects was often not in line with existing evidence. Vaginal discharge has been reported to occur in $25 \%$ of patients [20] and vaginal blood loss in 6-46\% of patients [20-22] at 1 year after treatment. A large proportion (57\%) of respondents considered (and possibly discussed with patients) that serious vaginal discharge occurs more frequently than it actually does. These discrepancies between perceived and actual complication rates may negatively affect the willingness of the gynecologist to propose and of the patient to undergo pessary treatment.

Large differences in follow-up intervals were reported by the respondents. Shorter intervals could be associated with unnecessary higher costs per patient. However, there is no clarity in the literature as to the ideal or minimal follow-up intervals after initial placement. Neither is there any evidence concerning the proportion of patients who are able to learn to clean and replace the pessary themselves. A majority of the respondents reported that they routinely instruct patients about self-management, including how to change and clean the pessary at home. However, from this survey we cannot determine the success of this advice and training. 


\section{Conclusions}

This survey clearly showed that there is large variation in the use of pessaries in the management of POP. This includes the information patients are provided with. Other variations concern follow-up after placement. A prospective study regarding effectiveness and consequences of the use of pessaries in the management of POP, and patient satisfaction with this approach, is needed.

Acknowledgments We acknowledge the contribution of those who responded to the questionnaire.

\section{Conflicts of interest None.}

\section{Appendix 1}

Table 5 Full survey questionnaire

Topic 1: Characteristics of gynecologists and hospitals
A] In what type of department
do you work?

B] How many new patients with vaginal prolapse are seen in your clinic annually?

C] How many vaginal prolapse surgery cases are there in your clinic annually?

D] How many new patients with incontinence are seen in your clinic annually?

General hospital
Teaching hospital
Academic hospital
Private practice
$0-200$
$201-400$
$401-600$
$601-800$
$>800$
$0-100$
$101-150$
$151-200$
$201-300$
$>300$
$0-25$
$26-50$
$51-75$
$76-100$
$>100$
Yes
No
Yes
o but consensus
No

Topic 2: Selection of patients

$$
\begin{aligned}
& \text { A] Do you propose pessary } \\
& \text { placement as your standard } \\
& \text { initial treatment? }
\end{aligned}
$$

Table 5 (continued)

B] What type of prolapse or complaint is most suitable for pessary treatment (multiple-choice question)?

C] Does stage of prolapse influence pessary treatment?

D] Does patient age influence pessary treatment?

Topic 3: Follow-up management

A] What is the interval to the first follow-up after initial placement in weeks?

B] Which professional is responsible for the first follow-up visit after initial placement

C] Do the intervals between follow-up visits change after initial placement (multiple-choice question)?

D] Do you prescribe estrogens (oral or vaginal use)?

Prolapse anterior compartment Prolapse middle compartment Prolapse posterior compartment

Stress incontinence Urge incontinence

Constipation

Obstructed defecation

Yes

No

Yes

No

2weeks

3 weeks

4weeks

6weeks

8 weeks

12 weeks

16weeks

Same caregiver

Same caregiver, later on general practitioner

Same caregiver or general practitioner

A specialist nurse

Patient wishes

Same interval continued

Shorter intervals if complaints

Longer intervals if no complaints

Yes

When indicated (vaginal atrophy)

When indicated (other than atrophy) No

Topic 4: Information gynecologists provides to patients including the option of self-management

A] How often does vaginal discharge occur due to $5-20 \%$ pessary treatment for $20-40 \%$ prolapse?

$40-60 \%$

$60-75 \%$

$>75 \%$

B] How often does vaginal blood $\quad 5-20 \%$ loss occur due to pessary $\quad 20-40 \%$ treatment for prolapse? $\quad 40-60 \%$

$60-75 \%$

$>75 \%$

$\begin{array}{cc}\text { C] What is the average chance } & 0-25 \% \\ \text { on getting surgical treatment } & 25-50 \%\end{array}$ for prolapse after pessary $\quad 50-75 \%$ treatment? $\quad 75-100 \%$

D] How often does pessary $\quad 5-15 \%$ extrusion occur? $\quad 15-30 \%$

$30-50 \%$

$>50 \%$

$5-20 \%$

$20-50 \%$

$>50 \%$
Not in some cases

No 
Table 5 (continued)

\begin{tabular}{cl} 
F] Do you give advice on & Always \\
self-management? & Regularly \\
& No \\
G] Do you give instructions & Always \\
on self-management? & Regularly \\
& No \\
\hline
\end{tabular}

Open AccessThis article is distributed under the terms of the Creative Commons Attribution 4.0 International License (http:// creativecommons.org/licenses/by/4.0/), which permits unrestricted use, distribution, and reproduction in any medium, provided you give appropriate credit to the original author(s) and the source, provide a link to the Creative Commons license, and indicate if changes were made.

\section{References}

1. Olsen AL, Smith VJ, Bergstrom JO, Colling JC, Clark AL (1997) Epidemiology of surgically managed pelvic organ prolapse and urinary incontinence. Obstet Gynecol 89(4):501-506. doi:10. 1016/S0029-7844(97)00058-6

2. Maher C, Feiner B, Baessler K, Schmid C (2013) Surgical management of pelvic organ prolapse in women. Cochrane Database Syst Rev 4, CD004014. doi:10.1002/14651858.CD004014.pub5

3. Bugge C, Adams EJ, Gopinath D, Reid F (2013) Pessaries (mechanical devices) for pelvic organ prolapse in women. Cochrane Database Syst Rev 2, CD004010. doi:10.1002/14651858. CD004010.pub3

4. Hagen S, Stark D, Maher C, Adams E (2006) Conservative management of pelvic organ prolapse in women. Cochrane Database Syst Rev 4, CD003882. doi:10.1002/14651858.CD003882.pub3

5. Nygaard I, Barber MD, Burgio KL et al (2008) Prevalence of symptomatic pelvic floor disorders in US women. JAMA 300(11):13111316. doi:10.1001/jama.300.11.1311

6. Swift S, Woodman P, O'Boyle A et al (2005) Pelvic Organ Support Study (POSST): the distribution, clinical definition, and epidemiologic condition of pelvic organ support defects. Am J Obstet Gynecol 192(3):795-806. doi:10.1016/j.ajog.2004.10.602

7. Komesu YM, Rogers RG, Rode MA et al (2008) Patient-selected goal attainment for pessary wearers: what is the clinical relevance? Am J Obstet Gynecol 198(5):577.e1-577.e5

8. Robert M, Schulz JA, Harvey MA, et al (2013) Technical update on pessary use. J Obstet Gynaecol Can 35(7):664-74
9. Lamers BHC, Broekman BMW, Milani AL (2011) Pessary treatment for pelvic organ prolapse and health-related quality of life: a review. Int Urogynecol J 22(6):637-644. doi:10.1007/s00192-011-1390-7

10. Ko PC, Lo TS, Tseng LH, Lin YH, Liang CC, Lee SJ (2011) Use of a pessary in treatment of pelvic organ prolapse: quality of life, compliance, and failure at 1-year follow-up. J Minim Invasive Gynecol. 18(1):68-74. doi:10.1016/j.jmig.2010.09.006.

11. Geoffrion R, Zhang T, Lee T, Cundiff GW (2013) Clinical characteristics associated with unsuccessful pessary fitting outcomes. Female Pelvic Med Reconstr Surg 19(6):339-345. doi:10.1097/ SPV.0b013e3182a26174

12. Clemons JL, Aguilar VC, Tillinghast TA, Jackson ND, Myers DL (2004) Risk factors associated with an unsuccessful pessary fitting trial in women with pelvic organ prolapse. Am J Obstet Gynecol 190(2):345-350. doi:10.1016/j.ajog.2003.08.034

13. Lone F, Thakar R, Sultan AH, Karamalis G (2011) A 5-year prospective study of vaginal pessary use for pelvic organ prolapse. Int J Gynaecol Obstet 114(1):56-59. doi:10.1016/j.ijgo.2011.02.006

14. Maito JM, Quam ZA, Craig E, Danner KA, Rogers RG (2006) Predictors of successful pessary fitting and continued use in a nurse-midwifery pessary clinic. J Midwifery Womens Health 51(2):78-84. doi:10.1016/j.jmwh.2005.09.003

15. Bugge C, Hagen S, Thakar R (2013) Vaginal pessaries for pelvic organ prolapse and urinary incontinence: a multiprofessional survey of practice. Int Urogynecol J 24(6):1017-1024. doi:10.1007/ s00192-012-1985-7

16. Gorti M, Hudelist G, Simons A (2009) Evaluation of vaginal pessary management: a UK-based survey. J Obstet Gynaecol 29(2): 129-131. doi:10.1080/01443610902719813

17. Friedman S, Sandhu KS, Wang C, Mikhail MS, Banks E (2010) Factors influencing long-term pessary use. Int Urogynecol J 21(6): 673-678. doi:10.1007/s00192-009-1080-x

18. Mutone MF, Terry C, Hale DS, Benson JT (2005) Factors which influence the short-term success of pessary management of pelvic organ prolapse. Am J Obstet Gynecol 193(1):89-94. doi:10.1016/j. ajog.2004.12.012

19. Yamada T, Matsubara S (2011) Rectocoele, but not cystocoele, may predict unsuccessful pessary fitting. J Obstet Gynaecol 31(5):441442. doi:10.3109/01443615.2011.577253

20. Sarma S, Ying T, Moore KH (2009) Long-term vaginal ring pessary use: discontinuation rates and adverse events. BJOG 116(13):17151721. doi:10.1111/j.1471-0528.2009.02380.x

21. Fernando RJ, Thakar R, Sultan AH, Shah SM, Jones PW (2006) Effect of vaginal pessaries on symptoms associated with pelvic organ prolapse. Obstet Gynecol 108(1):93-99. doi:10.1097/01. AOG.0000222903.38684.cc

22. Broens-Oostveen M, Mom R, Lagro-Janssen A (2004) De genitale prolaps; behandeling en beloop in vier huisartspraktijken. Ned Tijdschr Geneeskd 148(29):1444-1448 